\title{
O processo de integração regional como um jogo de coordenação: uma análise comparada para o setor hidroelétrico da ECOWAS, SADC, CAN e MERCOSUL
}

\author{
The regional integration process as a coordination \\ game: a comparative analysis for the hydroelectric \\ sector of ECOWAS, SADC, CAN and MERCOSUR
}

DOI: $10.21530 /$ ci.v15n1.2020.933

Taiane Las Casas Campos ${ }^{1}$

Fátima Anastasia ${ }^{2}$

\section{Resumo}

Neste artigo são analisados comparativamente quatro processos de integração regional de países em desenvolvimento: Comunidad Andina de Naciones (CAN), Mercado Comum do Sul (MERCOSUL), Southern African Development Community (SADC) e Economic Community of West African States (ECOWAS). Argumenta-se que as negociações regionais se pautam na consolidação de agendas, para as quais a estratégia dominante dos atores é a cooperação. Tais processos ocorrem por meio dos jogos de coordenação, com vistas a definir um "ponto de coordenação” entre as preferências dos Estados-membros dos acordos regionais. Discute-se a conformação dos acordos regionais para o tema da energia elétrica, nos quatro casos, visando demonstrar que as preferências dos Estados-membros acabam por estabelecer diferentes formatos relativamente à integração regional e à regulação estatal. Seu objeto central é a integração regional e suas variações. O tema da energia elétrica ${ }^{3}$ entra apenas como referente empírico para a análise em tela.

Palavras-chave: Integração Regional; Jogos de Coordenação; Países em Desenvolvimento; Energia.

1 Possui mestrado em Teoria Econômica pela Universidade Federal de Minas Gerais (1998); doutorado em Administração pela Universidade Federal de Minas Gerais (2003) e pós-doutorado pelo IUPERJ. Atualmente é professor adjunto IV do Departamento de Relações Internacionais- Programa de Pós-Graduação Relações internacionais da Pontifícia Universidade Católica de Minas Gerais. ORCID: https://orcid.org/0000-0002-2740-0640; email: lascasas@pucminas.br

2 Possui graduação em Comunicação Social pela Pontifícia Universidade Católica de Minas Gerais (1977), mestrado em Ciência Política pela Universidade Federal de Minas Gerais (1985) e doutorado em Ciência Política (Ciência Política e Sociologia) pelo Instituto Universitário de Pesquisas do Rio de Janeiro (1992). Atualmente é professora adjunto IV do Departamento de Relações Internacionais da Pontifícia Universidade Católica de Minas Gerais. ORCID: https://orcid.org/0000-0001-5839-9155; email: fatima.anastasia@gmail.com.

3 Ressalte-se que, neste artigo, a menção ao tema energia refere-se sempre à energia elétrica.

Artigo submetido em 04/04/2019 e aprovado em 05/03/2020. 


\begin{abstract}
In this article four regional integration processes of developing countries are comparatively analyzed: Andean Community (CAN), Southern Common Market (MERCOSUR), Southern African Development Community (SADC) and Economic Community of West African States (ECOWAS). It is argued that regional negotiations are based on the consolidation of agendas, for which the actors' dominant strategy is cooperation. Such processes occur through coordination games, with a view to defining a "point of coordination" between the preferences of the member states of the regional agreements. The conformation of regional agreements regarding electric energy is discussed in the four cases, aiming to demonstrate that the preferences of the Member States end up establishing different formats in relation to regional integration and state regulation. Its central object is regional integration and its variations. The electric energy theme is only an empirical reference for the analysis on screen.
\end{abstract}

Keywords: Regional Integration; Coordination Games; Developing Countries; Energy.

\title{
Introdução
}

Como variam os acordos regionais celebrados por países em desenvolvimento? Esta é a pergunta que informa a construção deste artigo. As pesquisas empíricas, bem como as proposições teóricas acerca do processo de integração regional, são, ainda, a despeito do crescente número de casos, uma das grandes lacunas no campo das Relações Internacionais. O paradigmático caso europeu tem fundamentado tanto as proposições teóricas quanto a prescrição de políticas para a integração, o que acaba por conferir a esse processo condição de parâmetro para a construção de outros processos de integração regional (Schmitter 2010).

Sabe-se, no entanto, que nem sempre as teorias de integração regional, construídas a partir do caso europeu, viajam bem (Malamud 2011). As especificidades de processos de integração ocorridos em outras regiões não são apreendidas adequadamente por teorias eurocêntricas e variáveis importantes para a explicação de tais processos podem estar ausentes de tais modelos, como, por exemplo, a variável sistema de governo, crucial para o entendimento da configuração imprimida ao MERCOSUL (Malamud 2011).

A proposição central deste artigo é a de que processos de integração regional devem ser analisados tendo por referência a lógica que informa os problemas de ação coletiva (Kurrild-Klitgaard 1997) referentes a temas de interesse dos paísesmembros. Agendas como preservação ambiental, integração energética, segurança 
pública e externa, comércio, dentre outras, trazem aos Estados diferentes dilemas de ação coletiva. No que se refere ao objeto deste artigo, o dilema em tela relaciona-se às dificuldades e, no limite, à impossibilidade de cada país prover o bem público, energia elétrica, especialmente de fonte hídrica, sem o recurso à cooperação. A superação desses dilemas significa que os Estados viabilizaram a cooperação e, como resultado, celebraram acordos formais relativos a essas agendas. Assim, o processo de integração regional é o somatório de acordos formais referentes a cada uma dessas agendas.

A estratégia analítica escolhida no âmbito deste artigo privilegiou a análise comparada dos quatro agrupamentos, uns com os outros, em detrimento de descrições pormenorizadas de cada um desses blocos, tendo em vista os objetivos de, a partir de parâmetros bem definidos, realizar uma comparação sistemática que permita identificar semelhanças e diferenças nas estruturas institucionais desenvolvidas pelos quatro blocos, com vistas a construir acordos referentes à agenda de energia elétrica, a partir de condições de acesso muito distintas a esse recurso. Por este motivo, o artigo toma como referencial empírico, exclusivamente, os documentos formais que resultam do processo decisório voltado para a integração regional no setor da energia elétrica. O que interessa analisar é a capacidade estatal construída, a partir da celebração de tais acordos e tratados, de institucionalizar, no nível regional, a cooperação entre os Estados sob análise.

Vale ressaltar ainda que, por contraste com a integração europeia, que tem avançado na construção de instituições supranacionais e multitemáticas, como a Comissão Europeia e o Parlamento Europeu, os processos de integração examinados neste artigo se traduzem em instituições intergovernamentais e recortadas em áreas temáticas específicas. A construção de um eficiente e moderno setor de infraestrutura é uma agenda importante para países em desenvolvimento, tanto internamente quanto no âmbito regional. Nos últimos anos, as elevadas taxas de crescimento econômico obtidas por muitos países desses blocos incrementaram a demanda por energia, sem o correspondente aumento da oferta. Contudo, o aspecto comum ao setor de infraestrutura se refere aos elevados níveis de investimento, sua demanda por escala, seja de produção ou consumo e, acima de tudo, sua importância estratégica para o desenvolvimento.

O artigo contém quatro seções, além desta introdução e das considerações finais. A primeira apresenta o modelo de análise utilizado. A segunda descreve a configuração dos blocos em termos de estruturas institucionais responsáveis pelos 
processos decisórios. A terceira seção examina as estruturas regionais relativas à agenda de energia. A conjugação dessas duas últimas seções permite analisar, na quarta seção, similaridades e diferenças entre esses blocos e as relações entre as particularidades de cada um deles e os designs específicos, encontrados em cada bloco, para a integração no setor de energia elétrica. As considerações finais apontam os achados deste artigo e sugerem novos desdobramentos desta agenda de pesquisa.

\section{Integração Regional e Dilemas de Ação Coletiva}

Processos de integração regional envolvem necessariamente dilemas de ação coletiva. As agendas levadas às mesas de negociação são traduções dos interesses/preferências dos países membros, relativamente a cada tema em discussão, bem como de seus recursos materiais e políticos, os quais - interesses/ preferências e recursos - são apresentados e confrontados no âmbito do processo decisório relativo a cada issue-area. Inicialmente, poder-se-ia supor a presença de similaridades socioeconômicas e políticas entre países em desenvolvimento do continente sul-americano e da África Subsaariana e, daí, derivar a existência de semelhanças nos processos de integração regional da Comunidad Andina de Naciones $^{4}$-CAN-, do Mercado Comum do Sul - MERCOSUL ${ }^{5}$-, da Southern African Development Community ${ }^{6}$ - SADC - e da Economic Community of West African States ${ }^{7}$ - ECOWAS - no que se refere aos tópicos a serem negociados e à evolução desse processo para cada bloco.

No entanto, em se tratando de interconexão hidrelétrica, empiria em tela, pode-se observar a presença de diferenças marcantes entre os países membros dos diferentes blocos. A tabela 1 mostra as variações no acesso à eletricidade que atravessam esses países. Tais variações justificam a escolha dessa empiria - a interconexão hidrelétrica - para a averiguação das diferenças entre os processos de integração regional aqui estudados.

4 Bolívia, Colômbia, Equador e Peru.

5 Argentina, Brasil, Paraguai, Uruguai e Venezuela.

6 África do Sul, Angola, Botsuana, República Democrática do Congo, Lesoto, Madagascar, Malawi, Maurícia, Moçambique, Namíbia, Suazilândia, Tanzânia, Zâmbia e Zimbábue.

7 Benim, Burkina Faso, Cabo Verde, Costa do Marfim, Gâmbia, Gana, Guiné-Bissau, Guiné, Libéria, Mali, Níger, Nigéria, Senegal, Serra Leoa e Togo. 
Tabela 1 - Acesso à eletricidade (\% da população) ${ }^{8}$

\begin{tabular}{c|c|c|c|c}
\hline Ano & Ecowas & Sadc & Can & Mercosul \\
\hline 1990 & 12,48 & 23,65 & 73,86 & 90,44 \\
1995 & 18,32 & 27,53 & 77,97 & 91,52 \\
2000 & 25,61 & 31,88 & 82,77 & 95,02 \\
2005 & 29,02 & 36,40 & 84,52 & 97,10 \\
2010 & 34,07 & 40,69 & 91,67 & 98,59 \\
2015 & 41,50 & 46,16 & 95,60 & 99,64 \\
2016 & 43,65 & 47,81 & 96,71 & 99,60 \\
\hline
\end{tabular}

Fonte: Elaborado pelas autoras com dados do World Bank. n.d.

Argumenta-se, aqui, que as diferenças de acesso à eletricidade (Tabela 1) decorrem, fundamentalmente, de variações nas capacidades estatais dos países membros de tais agrupamentos. Segundo Evans (1993), capacidade estatal é a capacidade de ação do Estado 9 . Interessam a este artigo, especialmente, as capacidades fiscal/alocativa ${ }^{10}$ e administrativa/de implementação ${ }^{11}$. Os países com menores capacidades estatais para a provisão independente dos serviços de energia para as suas populações terão interesses/preferências mais intensas por soluções regionais desses problemas, ainda que renunciando ao exercício de regulação estatal da provisão de tais serviços. Por consequência, o problema será tratado no âmbito dos agrupamentos regionais, cujas instituições ficarão responsáveis pelo controle e acompanhamento da provisão do serviço por atores do mercado.

Não obstante, apesar das diferenças entre os membros desses acordos regionais relativamente ao acesso de suas populações à eletricidade, eles enfrentam os mesmos problemas de ação coletiva. A escassez de tais recursos, nos países membros do ECOWAS e do SADC, expressiva da baixa capacidade desses Estados em resolvê-la, agudiza tais problemas, colocando o tema do acesso a recursos energéticos em um ponto elevado na hierarquia de prioridades dos decisores desses agrupamentos.

8 O Banco Mundial não especifica, na base de dados, quais as fontes de energia foram consideradas para compor a variável "acesso a eletricidade". A definição dessa variável na base de dados é "is the percentage of population with access to electricity. Electrification data are collected from industry, national surveys and international sources”. (World Bank n.d.)

9 Há uma extensa literatura sobre capacidades estatais. Ver, a respeito, Cigolani 2013.

10 Fiscal Capacity emphazises the state's power to extract resources from the society, mainly in the form of tax. Occasionally, it also refers to the eficiency of government spending (e. g. Dincecco 2011) (Cingolani 2013, 28).

11 The administrative capacity of the state is rooted in the Weberian tradition regarding the modern state and the existence of a professional and insulated bureaucracy (e. g. Weber 1978). (Cingolani 2013, 28). 
Problemas de ação coletiva são objetos da “Teoria dos Jogos” (Martin 1992, 1998; Mello 1997), verificando-se duas situações clássicas para definir esses dilemas: jogos de coordenação e jogos de colaboração. No primeiro caso, supõese que atores preferem cooperar, sendo essa a estratégia dominante. Como suas preferências não são coincidentes, a cooperação é resultado da barganha política visando converter um "eixo de coordenação" em um "ponto de coordenação", ou seja, na celebração do acordo (Snidal 1985; Stein 1982). Nos jogos de colaboração, do tipo dilema do prisioneiro, relativos a agendas como comércio e segurança, as preferências dos atores são pela não cooperação e a celebração dos acordos só se torna factível se houver incentivos seletivos (Olson 1999) que alterem esse comportamento, seja pela punição aos dissidentes, seja pela oferta de recompensas.

Se o problema de ação coletiva é similar, as estruturas institucionais desenhadas para enfrentá-lo podem ser diferentes. Conforme mencionado, o caso europeu tem sido considerado um paradigma em função das estruturas institucionais criadas. Muitas vezes, a não observância desse "modelo" tem sido interpretada como "falha" no processo de integração. Defende-se, neste artigo, que essas estruturas variam, para cada agenda e para cada bloco, conforme os interesses dos países membros, a partir de suas condições históricas, econômicas, políticas e sociais.

A ratificação de um acordo regional sobre eletricidade pode dar a segurança jurídica necessária para que empresas públicas e privadas, governos e demais atores invistam em projetos transfronteiriços, de forma a possibilitar, por exemplo, que a eletricidade gerada em um país seja consumida em outro, que haja acesso irrestrito de pessoas e empresas ao território alheio, compatibilidade técnica entre geração e distribuição, dentre outros, sendo necessária, para sua efetivação, a celebração, pelos participantes, de acordos formais.

Essa configuração do problema de ação coletiva permite avaliar as contribuições e o formato que as instituições regionais apresentam para a cooperação entre Estados, especificamente no setor hidrelétrico. O primeiro aspecto institucional a ser analisado se refere aos mecanismos que contribuiriam para alcançar o ponto de coordenação dos participantes. Como a estratégia dominante é cooperar, a estrutura institucional precisa, sobretudo, prover informações aos Estados. Snidal (1985) considera ser a convenção o design ideal para equacionar o jogo de coordenação e afirma que a estrutura deve ter baixos níveis de institucionalização, já que suas funções básicas visam facilitar as escolhas, interpretações e a observância de uma convenção particular. Além disso, essa estrutura deve prover informações sobre preferências e políticas dos Estados e ser um fórum para a resolução dos 
problemas de barganha. Não há, assim, incentivos para criação de instituições supranacionais ou de mecanismos de enforcement, já que, alcançado o ponto de convergência das preferências, nenhum ator tem motivações para desertar.

Cabe ressaltar, ainda, seguindo Martin e Simmons (1998), que instituições internacionais são simultaneamente causa e efeito das escolhas dos Estados. Os Estados escolhem o design institucional que melhor se adequa à solução de seus dilemas de ação coletiva. Uma vez escolhidas, as instituições moldarão seus comportamentos (Martin e Simmons 1998). O design institucional é, portanto, o resultado de escolhas dos Estados nas agendas de cooperação.

Essa adequação do design institucional às preferências dos atores pode levar os Estados a formalizarem diferentes tipos de acordos internacionais: memorandos de entendimento, acordos-quadro, protocolos ou tratados. O formato a ser utilizado depende das preferências e, mais importante, dos estágios de negociação ao longo do “eixo de coordenação”. Um memorando de entendimento pode ser o formato institucional possível e desejado pelos atores diante de preferências dadas. Em contrapartida, é possível que um tratado, que implica maior grau de compromisso dos participantes, seja ratificado nas fases iniciais das negociações.

A partir desses elementos, o argumento ora desenvolvido reside na compreensão de que as agendas de saúde, educação, infraestrutura em geral, e eletricidade em particular, são claramente configuradas nos moldes de um jogo de coordenação, sendo o processo de negociação política elemento essencial para fazer convergirem as preferências dos participantes para um determinado ponto de coordenação. Essas preferências determinam as estruturas institucionais constituídas por cada um dos blocos que, por um lado, negociam os termos dos acordos e por outro dão prosseguimento na condução e consolidação da agenda.

Vale ressaltar, ainda, que as preferências - e os recursos - dos atores em interação estratégica estão relacionados a dois diferentes, ainda que entrelaçados, eixos de coordenação. O primeiro se refere ao grau de institucionalização que tais atores estão dispostos a conferir à cooperação entre eles, em cada agenda específica, mais institucionalização significando mais regulação pelo Estado. E, inversamente, menos institucionalização significando maior escopo para a ação não regulada das forças de mercado. O segundo eixo se refere ao grau de regionalização/ harmonização da política pública objeto da cooperação, o que implica consideração obrigatória do conceito de soberania, que, segundo Locke (1978), é absoluta, porém divisível em áreas. Tal questão, então, remete ao desafio de encontrar, para cada agrupamento regional, o ponto de coordenação que expresse a combinação 
adequada, para cada contexto, entre o grau de institucionalização e o grau de regionalização acordados para o tema da energia elétrica. A figura 1 apresenta graficamente o modelo de análise aqui desenvolvido:

Figura 1 - Modelo de análise

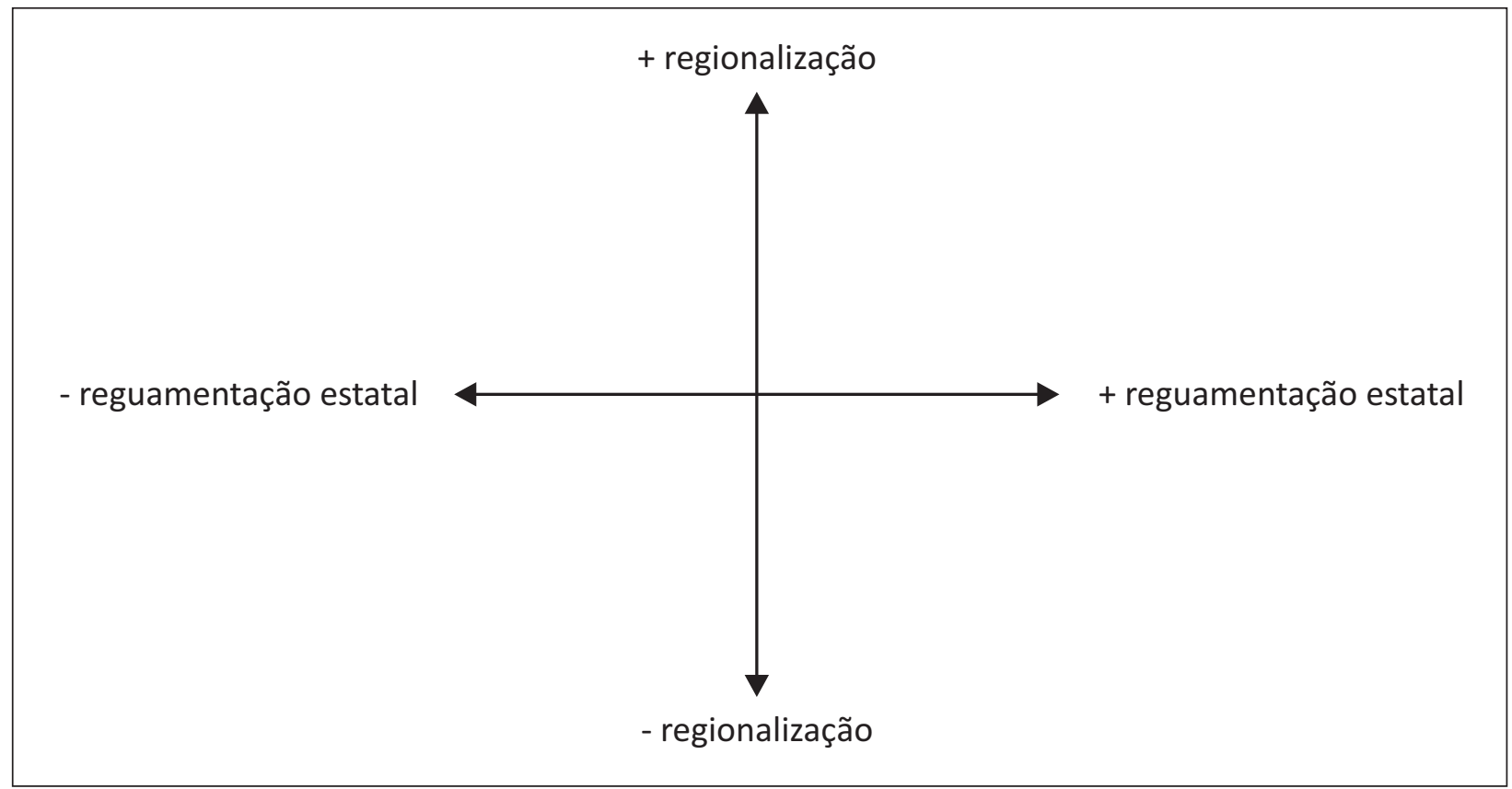

Fonte: Elaboração das autoras

\section{Estruturas Institucionais e processos decisórios}

As posições dos países nos eixos institucionalização e regionalização/ harmonização da política pública serão utilizadas como parâmetros para a análise comparativa em tela, considerando o ponto de coordenação que viabiliza a cooperação. No eixo institucionalização, as variações serão observadas a partir das seguintes categorias analíticas: 1) agências regionais com poder de agenda, ou seja, aquelas constituídas, em geral, por Chefes de Estado e/ou de governo, no primeiro nível, e por ministros de Relações Exteriores, no segundo nível ${ }^{12}$; 2) agências regionais de negociação entre os países membros, ou seja, aquelas constituídas, em geral, por ministros da área temática específica, no caso a de eletricidade.

120 arranjo institucional do MERCOSUL é a exceção relativamente a esta regra já que, geralmente, as reuniões das agências do primeiro nível são compostas por ministros das Relações Exteriores e as do segundo nível por ministros da Fazenda e presidentes de Bancos Centrais. Não obstante, os chefes de Estado podem se reunir ou participar destas agências e determinar as agendas em discussão. 
Os graus de institucionalização das agências e das regras decisórias variam entre os agrupamentos investigados. Relativamente:

1) às agências regionais com poder de agenda, interessa verificar o protagonismo de Chefes de Estado e de governo: maior a presença de chefes de Estado e de governo, maior o grau de institucionalização;

2) às agências regionais de negociação, mais específica a estrutura de negociação, para cada agenda, maiores as chances de incremento nos graus de regionalização/harmonização da política.

Seguem-se a descrição e a análise de cada agrupamento regional à luz dessas categorias analíticas. Ao final desta seção, será realizada a comparação entre os graus de institucionalização dos agrupamentos relativamente à política estudada.

No acordo constitutivo da Ecowas (1975), a instância máxima decisória é a Autoridade de Chefes de Estado e Governo, seguida pelo Conselho de Ministros, a Secretaria Executiva, o Tribunal da Comunidade e quatro comissões técnicas especializadas: 1. Comércio, aduana e pagamentos; 2 . Agricultura, indústria e recursos naturais; 3. transporte, telecomunicações e energia e 4. questões sociais e culturais.

Essa estrutura se manteve até 1996, quando os membros da ECOWAS redefiniram as comissões especializadas, estabelecendo, assim, as prioridades do bloco. Destaque-se a comissão de agricultura, indústria e recursos naturais que agora se divide em duas: uma para tratar de alimentação e agricultura e outra para questões relativas à indústria, ciência, tecnologia e energia. Ressalte-se que o tratado de 1975 e sua revisão (1993) detalham os compromissos assumidos pelos Estados membros relativamente à cooperação nessas agendas e que essas comissões especializadas tratariam de operacionalizar os compromissos assumidos naqueles tratados (Ecowas 1993). Assim, para cada área temática foi designada uma comissão e definido um conjunto de compromissos assumidos. No caso da energia, tem-se a Reunião de Ministros de Energia, instância de monitoramento e proposição de ações cooperativas (Ecowas 2003).

Conforme estabelecido no acordo, cada Comissão deverá submeter suas propostas ao Conselho de Ministros e, mais importante, "assegurar a harmonização e coordenação de políticas nacionais e a promoção de programas, projetos e atividades de integração” (Ecowas 199 3, 5) ${ }^{13}$. Em 2007, a Secretaria Executiva foi transformada na Comissão da ECOWAS, composta por departamentos responsáveis

13 (...) ensure the harmonisation and co-ordination of national policies and the promotion of integration programmes, projects and activities (...). 
pelas áreas temáticas definidas no acordo constitutivo do bloco e sua revisão, sendo um desses departamentos o de energia, responsável por fornecer a expertise técnica em energia e pelo desenho e implementação de projetos técnicos para a região, conforme decidido pelo Presidente (Ecowas, n.d.) Esse departamento disponibiliza informações sobre projetos de energia em execução entre os países membros e estabelece prioridades a serem avaliadas.

Além dessa estrutura institucional, os membros da ECOWAS criaram, nos anos 1980 e 1990, diversas agências especializadas, duas delas relativas ao setor de energia: West African Power Pool (WAPP - 1999), ECOWAS Regional Centre for Renewable Energy and Energy Efficiency (ERERA - 2008). Essas agências compõem a estrutura do bloco e, portanto, têm suas regras, princípios e metas definidos pelo Conselho de Ministros da ECOWAS. O ministro encarregado dos assuntos da ECOWAS do país cujo chefe de Estado foi nomeado presidente da Autoridade de Chefes de Estado e Governo é automaticamente o Presidente do Conselho de Ministros, da Comissão e preside as demais reuniões estatutárias do bloco, como os comitês técnicos.

A estrutura institucional da SADC é composta por: Cúpula de chefes de Estado e governo, que se reúne pelo menos uma vez por ano; Órgão sobre Política, Defesa e Segurança, o Tribunal da SADC; Conselho de Ministros (relações exteriores, economia e correlatos) responsável pela supervisão e monitoramento da agenda regional; e Comitês Ministeriais Setoriais, compostos pelos ministros dos países membros, nas respectivas áreas temáticas, sendo uma delas a de Infraestrutura e Serviços, que trata da energia. Finalmente, tem-se a Secretaria Executiva, cujas funções são propor, planejar e executar as agendas da SADC. Seu Secretário Executivo Adjunto tem a função de promover, executar e avaliar os acordos regionais relativos à infraestrutura em geral e à energia em particular (Sadc, n.d.). Esse Secretário Executivo Adjunto é responsável pela Diretoria de Infraestrutura e Diretoria de Serviços, e é o "presidente" da Southern African Power Pool- SAPP-, criada em 1995, que atualmente é composta por 16 empresas, estatais e privadas, que geram e distribuem energia para os países da SADC. Mensalmente, essa agência reporta as condições do mercado regional de energia, com os volumes e preços comercializados.

Na América do Sul, o MERCOSUL foi constituído em 1991, por Argentina, Brasil, Paraguai e Uruguai. A Venezuela aderiu ao bloco em 2011 e a Bolívia está em fase de ascensão ao bloco. A estrutura institucional do bloco é composta por: 1. Conselho do Mercado Comum - CMC - órgão máximo de decisão, composto 
pelos ministros das relações exteriores dos países membros; 2. Grupo do Mercado Comum - GMC - órgão decisório executivo, responsável por fixar os programas de trabalho, e por negociar acordos com terceiros em nome do bloco, por delegação expressa do CMC. O GMC se pronuncia por Resoluções, e está integrado por representantes dos Ministérios de Relações Exteriores e de Economia, e dos Bancos Centrais dos Estados Parte; 3. Comissão de Comércio do MERCOSUL; 4. Comissão Parlamentar Conjunta; 5. O Foro Consultivo Econômico Social; 6. Comissão de Representantes Permanentes do MERCOSUL (CRPM), 7. Secretaria do MERCOSUL.

Tanto o CMC quanto GMC contam com instâncias especializadas em diferentes agendas. Por exemplo, a Reunião de Ministros de Minas e Energia é vinculada ao CMC e os Sub- Grupos de trabalho vinculados ao GMC, este último composto por especialistas nas áreas temáticas em discussão.

A estrutura institucional da CAN (1969) é composta por: Conselho Presidencial Andino; o Conselho Andino de Ministros de Relações Exteriores; a Comissão da Comunidade Andina; a Secretaria Executiva; o Tribunal, o Parlamento, dentre outros. A Comissão Andina é composta por um representante de cada país e trata essencialmente das questões relativas ao comércio entre os membros.

A proposição e o acompanhamento das iniciativas no âmbito regional são realizados pela Comissão e pela Secretaria Geral, que podem propor a criação de comitês e conselhos, compostos por especialistas, dos governos ou não, de forma a ter assessoria técnica. Os Comitês Assessores são compostos por ministros ou secretários de Estado dos países membros, têm caráter permanente e subsidiam o Conselho de Ministros de Relações Exteriores, a Comissão e a Secretaria. Por fim, a Secretaria pode constituir Grupos Assessores ad hoc ou Reunião de Especialistas, de caráter temporário, para subsidiar suas decisões.

A estrutura de governança para o setor de energia da CAN é composta pelo Comité Andino de Organismos Normativos y Organismos Reguladores de Servicios de Electricidad - CANREL - , que propõe as normativas para o setor, a serem ratificadas pela Comissão da Comunidade Andina. A Comissão é composta por um representante de cada país (normalmente ministros de comércio ou de integração), cuja presidência é exercida pelo representante do país que exerce a presidência da CAN. Aprovada uma resolução, cabe à secretaria executiva da CAN criar as condições para implementar e monitorar o que foi acordado.

Considerando que a estratégia dominante dos Estados nas negociações de energia é a cooperação, e que as negociações se prestam a converter o eixo de coordenação em um ponto de coordenação, torna-se relevante analisar como os 
Estados, em um primeiro momento, decidem iniciar o processo de negociação e, em um segundo momento, estabelecem os termos dos acordos que dão prosseguimento na condução e consolidação da agenda.

Pode-se considerar que a decisão relativa a quais agendas serão negociadas é atribuição da instância máxima de cada um dos blocos. À exceção do MERCOSUL, nos demais blocos essa instância é composta por chefes de Estado e de governo, que se reúnem pelo menos uma vez ao ano.

Quadro 1 - Estruturas institucionais regionais voltadas à agenda de energia elétrica

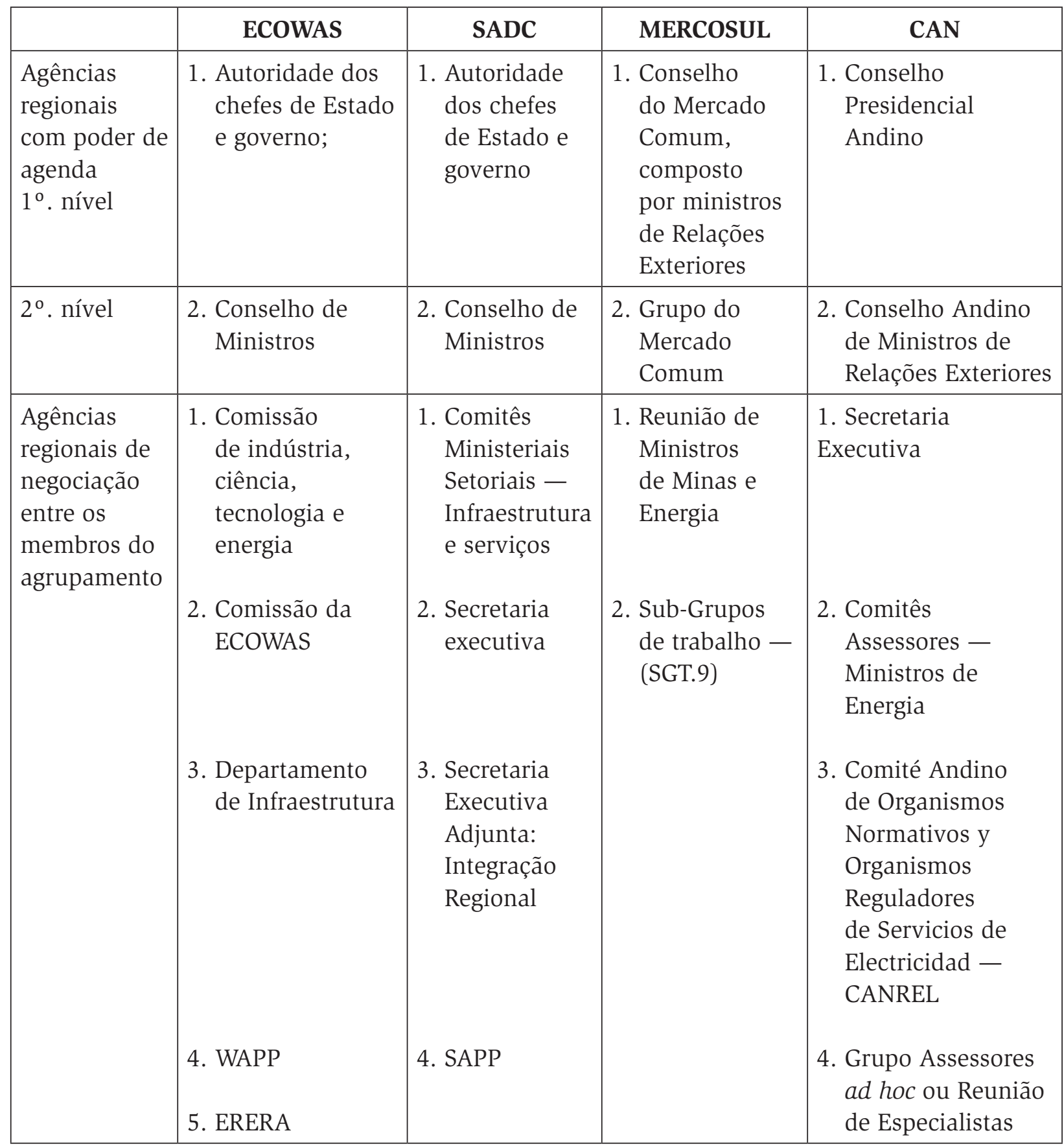

Fonte: Elaborado pelas autoras a partir de diversos documentos dos blocos referenciados no texto acima. 
A classificação dos graus de institucionalização será feita considerando-se três posições: ALTA, MÉDIA e BAIXA. O exame do Quadro 1 permite classificar os quatro agrupamentos no eixo respectivo da seguinte forma: ECOWAS, SADC e CAN contam com agências com poder de agenda formadas por chefes de Estado e de Governo e, no segundo nível, por ministros de Relações Exteriores. Porém, no MERCOSUL, as agências dos níveis um e dois são compostas por Ministros de Relações Exteriores.

Estabelecidas as agendas, é necessário que especialistas da área sejam convocados para iniciar o processo de negociação e construir as bases dos acordos. Nos quatro blocos existe essa instância institucional formada por especialistas na área de energia. Contudo, na ECOWAS e na SADC essa estrutura é permanente e está definida nos acordos constitutivos dos blocos. No MERCOSUL e na CAN essa instância está presente, mas tem caráter de assessoria às instâncias superiores, sendo que, no MERCOSUL, as reuniões podem (porém não são compulsórias) ocorrer por ocasião das reuniões do CMC e, na CAN, quando solicitadas. Vale sublinhar que essas instâncias são compostas pelos ministros de Minas e Energia dos países membros. Novamente, à exceção do MERCOSUL, os demais blocos têm uma Secretaria Executiva com poder de agenda, a despeito do fato de que também executam e monitoram o desenvolvimento da agenda.

Na ECOWAS e na SADC, a institucionalização da agenda é mais efetiva na medida em que, subordinados à Secretaria Executiva (Comissão da ECOWAS), encontram-se o Departamento de Infraestrutura (ECOWAS) e a Secretaria Executiva Adjunta: Integração Regional (SADC). Suas funções são propor, monitorar e viabilizar a implantação de acordos regionais de energia elétrica. Por fim, nesses dois blocos há também as organizações - WAPP e SAPP - que agregam as empresas de energia públicas e privadas dos países membros, viabilizando o monitoramento do setor. Observe-se que ambas estão sujeitas às regras da ECOWAS e da SADC e que seus presidentes são nomeados pelo Conselho de Ministros.

Portanto, considerando as características das agências regionais com poder de agenda e, também, aquelas agências de negociação, pode-se afirmar que ECOWAS e SADC apresentam ALTO grau de institucionalização, CAN apresenta MÉDIO grau de institucionalização e o MERCOSUL, BAIXO grau de institucionalização. 


\section{A agenda de energia nos blocos regionais}

À exceção da CAN, o mais antigo dos quatro blocos, os demais, já no início do processo de integração regional, contemplaram em suas negociações a agenda de energia.

Quadro 2 - Os acordos de energia

\begin{tabular}{|c|c|c|}
\hline Acordo Regional & Normativa para o setor de energia & Ano \\
\hline \multirow{5}{*}{ ECOWAS } & Energy Policy of ECOWAS & 1982 \\
\hline & Protocol of Energy & 1993 \\
\hline & Master Plan & 1999 \\
\hline & ECOWAS Energy Protocol & 2003 \\
\hline & Revised Master Plan & 2005 \\
\hline \multirow{3}{*}{ SADC } & Protocol of Energy & 1996 \\
\hline & SADC Energy Cooperation Plan and Strategies & 1996 \\
\hline & SADC Energy Action Plan & 1997 e 2000 \\
\hline \multirow{5}{*}{ MERCOSUL } & Diretrizes de política energética- GMC & 1993 \\
\hline & Pauta negociadora & 1996 \\
\hline & Memorando de entendimentos & 1998 \\
\hline & Reunião de Ministros & 2000 \\
\hline & Acordo-Quadro & 2005 \\
\hline \multirow{4}{*}{ CAN } & Marco General & 2002 \\
\hline & Suspensão do Marco Geral & 2009 \\
\hline & Autorização acordos bilaterais & 2011 \\
\hline & Marco Regulatório para a Interconexão Sub-regional de Energia & 2017 \\
\hline
\end{tabular}

Fonte: Elaborado pelas autoras a partir de diversos documentos dos blocos referenciados no texto abaixo.

Os membros da ECOWAS ratificaram seu Protocolo de Energia em 2003, cujo objetivo é extremamente claro e visa “[...] estabelecer um quadro legal para promover a cooperação no longo prazo no domínio da energia,... objetivando aumentar o investimento no sector da energia e aumentar o comércio de energia na região da África Ocidental"14 (Ecowas 2003, 9). O preâmbulo do protocolo se baseia no reconhecimento da necessidade de investimentos no setor. Assim,

14 establishes a legal framework in order to promote long-term co-operation in the energy field, ... with a view to achieving increased investment in the energy sector, and increased energy trade in the West Africa region" (Ecowas 2003, 9). 
o eixo central do Protocolo é estruturado com vistas a dar condições para que empresas multinacionais e investidores desenvolvam suas atividades nos países membros do bloco. Entre os oito capítulos do Protocolo, o primeiro trata das definições e do propósito do acordo, o segundo, mais longo, trata do comércio, definido como o compromisso de que "todos trabalharão para promover acesso ao mercado internacional de material de energia e produtos e equipamentos de energia relacionados em termos comerciais e, em geral, para desenvolver um mercado de energia aberto e competitivo" (Article 3, Ecowas, 2003). As cláusulas desse capítulo e do subsequente são relativas às garantias de trânsito de pessoas, materiais e produtos, promoção e proteção aos investimentos, compensação por perdas, expropriação, taxação, empresas estatais, dentre outras, visando atrair e dar garantias a empresas e investidores internacionais. Por fim, esse protocolo prevê a constituição e define as atribuições da Reunião de Ministros de Energia da ECOWAS, como a instância de implementação dos termos acordados no protocolo.

Observe-se que os membros da ECOWAS formularam planos de desenvolvimento do setor elétrico regional antes mesmo de ratificarem esse protocolo em 2003. Já em 1999, os ministros de energia entendiam que o setor deveria se pautar pelos mecanismos de mercado e propuseram: 1. um plano diretor para o desenvolvimento de instalações de produção de energia e interconexão de redes elétricas; 2 . criação de uma estrutura de coordenação para energia na região; 3. elaboração de um plano de ação para o desenvolvimento de projetos energéticos economicamente viáveis; 4. maior participação do setor privado no desenvolvimento de infraestruturas de produção e transmissão (Ecowas 1999). Como se verá, em linhas gerais, tais sugestões foram acatadas pela cúpula do bloco. A criação do West African Power Pool- WAPP aconteceu ainda em 1999 e sua estruturação, funcionamento e o reconhecimento como agência especializada da ECOWAS aconteceram em 2006.

Já o acordo constitutivo da SADC, de 1992, estabeleceu que seu principal objetivo é promover o desenvolvimento de seus membros, os quais acordaram cooperar em um amplo leque de agendas como segurança alimentar, agricultura, infraestrutura e serviços, ciência e tecnologia, dentre outros (Sadc 1991). Com essa diretriz, cinco anos depois, os membros da SADC ratificaram o Energy Protocol (1996). Os princípios gerais do protocolo explicitam a importância estratégica da energia para promover o desenvolvimento e viabilizar o crescimento econômico dos membros do bloco. O acordo se baseou no compromisso dos membros de se empenharem para harmonizar as políticas, estratégias e programas de energia da esfera nacional e regional e de cooperarem para desenvolver programas conjuntos 
de pesquisa, transferência de tecnologia, de recursos humanos e materiais entre os membros, além de buscar uma padronização no desenvolvimento e na aplicação de métodos comuns de utilização de energia. Ademais, e mais importante, os países membros se comprometeram em criar um ambiente propício para o setor privado participar integralmente no desenvolvimento do setor na região (Sadc 1996).

Para além desses compromissos genéricos, esse Protocolo tem seu eixo central (artigo 4) na formatação da estrutura institucional responsável por propor planos, programas e demais estratégias para a consolidação dos termos acordados, ou seja, harmonizar políticas, desenvolver tecnologias e recursos humanos e materiais para uma "política energética regional". Essa estrutura seria formada por uma Comissão, composta pelo Comitê de Ministros, um Comitê de Altos Funcionários e subcomitês técnicos e de ministros de energia dos países membros. Grande parte das cláusulas restantes do Protocolo se refere às funções e organização dessa estrutura institucional (Sadc, 1996).

Em dimensão mais pragmática, já em 1996, a SADC estabeleceu a SADC Energy Cooperation Policy and Strategy, que buscava definir parâmetros para a ação dos membros. Em 1997, o Departamento de Infraestrutura e Serviços da SADC elaborou o Energy Action Plan. Em 2001, ocorreu a refundação da SADC, com a repactuação de novas metas para o agrupamento e o Regional Indicative Strategic Development Plan - RISDP - (Sadc 2001). Esse plano contempla todas as áreas envolvidas na integração regional e traz metas e estratégias para cada uma delas, inclusive a de energia. Os projetos e metas para o setor de energia, alterados, foram incorporados em revisão do plano regional de desenvolvimento - Regional Infrastructure Development Master Plan - RIDMP - (Sadc 2012). Esse plano estava em elaboração desde 2007, quando a Cúpula de Chefes de Estado reconheceu a grave crise elétrica da região, ampliando as metas a serem atingidas, o que fundamentou o Energy Sector Plan, em 2012.

A Southern African Power Pool- SAPP (1995) - monitora as condições do mercado regional de energia. Ela é composta por 16 empresas estatais e privadas, que geram e distribuem energia para os países da SADC. Mensalmente, essa agência reporta as condições do mercado regional de energia, com os volumes e preços comercializados.

No âmbito do MERCOSUL foi ratificado, em 2005, um Acordo-Quadro, instrumento genérico que possibilita aos seus membros celebrarem acordos sub-regionais ou bilaterais definidos a partir dos parâmetros nele estabelecidos. Esse documento contém apenas três capítulos. O primeiro define o propósito de: 
“contribuir para avançar na integração energética regional em matéria de sistemas de produção, transporte, distribuição e comercialização nos Estados Partes, a fim de garantir os insumos energéticos e de gerar as condições para minimizar os custos das operações comerciais de intercâmbio energético entre os mencionados Estados” (Mercosul 2005).

O segundo capítulo trata da "Cooperação Regional” e prevê: 1) que as partes buscariam identificar atividades de intercâmbio, projetos e obras de infraestrutura energética a serem executadas de forma conjunta; 2) a execução de acordos regionais, sub-regionais ou bilaterais de interconexão no segmento de energia. Com esse formato, o acordo não prevê nenhum mecanismo ou instituição incumbido de operacionalizar ou propor alteração na estrutura do setor de energia na região. Ações nesse sentido seriam conduzidas de forma bilateral ou sub-regional pelos governos dos países interessados.

A conformação desse acordo se inicia em 1993, dois anos após a criação do MERCOSUL, quando foram aprovadas as Diretrizes de Políticas Energéticas no MERCOSUL (Mercosul 1993). As negociações dessas Diretrizes não lograram êxito, de forma que, em 1996, o Grupo do Mercado Comum aprovou Pautas Negociadoras do Subgrupo No 9 - Energias (Mercosur 1996). Em 1998, o Conselho de Mercado Comum (CMC) aprovou um memorando de entendimento, que traz as bases de um mercado regional de energia (Mercosul 1998). Em 2000, o CMC criou a Reunião de Ministros de Minas e Energia (Mercosur 2000), para propor os termos do Acordo-Quadro sobre Complementação Energética Regional, aprovado em 2005.

Não foram identificados projetos de cooperação energética regional entre os membros do MERCOSUL. Os projetos de cooperação são majoritariamente bilaterais, mas não fazem referência ou se enquadram nos termos do AcordoQuadro previamente estabelecido neste espaço de coordenação.

A CAN tem claramente explicitado o objetivo de construir um "Marco General para la Interconexión Subregional de Sistemas Eléctrico” visando organizar um mercado regional de eletricidade. Esses esforços se iniciaram em 2002, com a Resolução 536, que estabeleceu as regras para o intercâmbio de eletricidade entre os países membros, por meio de uma interconexão regional (Can 2002). Essas regras se referem à criação de mercado para o setor de energia na esfera sub-regional, com os compromissos de que os países membros "assegurariam as condições competitivas para o mercado de eletricidade, com preços e tarifas que reflitam os custos econômicos eficientes, evitando práticas discriminatórias e abusos da posição dominante" (Can 2002, 3). Os países membros se comprometeriam a 
alterar suas normativas nacionais, de forma a promover uma harmonização em matéria de operações de interconexões e de transações comerciais de eletricidade.

Essa resolução foi suspensa em 2009, e em 2011 a questão energética se torna regulada por acordos bilaterais ${ }^{15}$. Enfim, em 2017, diferentemente dos demais blocos analisados, os membros da CAN definiram um Marco Regulatório para a Interconexão Sub-regional de Energia, sinalizando a existência prévia de um mercado de energia e a necessidade de apenas criar as bases para o que denominam "Mercado Andino Elétrico Regional" (MAER), visando otimizar excedentes elétricos de cada país, aproveitar a complementaridade e a disponibilidade de recursos energéticos, o acesso livre, transparente e recíproco das informações do mercado e daquelas necessárias para o planejamento dos demais países. Essa Decisão da CAN estruturou o chamado "Mercado Andino Regional de Curto Prazo" para estabelecer as regras, ou seja, preços, (baseados nas curvas de oferta e demanda regional por energia), os operadores regionais, responsáveis por coordenar e viabilizar as Transações Internacionais de Eletricidade - TIR. Além disso, tal decisão estabelece que a secretaria geral da CAN deveria, mediante proposições da CANREL, elaborar o Regulamento Operativo, o Regulamento Comercial e o Regulamento de designações, funções e responsabilidades do Coordenador Regional.

Também no caso da CAN, há um longo processo de negociação com avanços e retrocessos visando um acordo regional de energia. No início dos anos 2000, delegados dos organismos reguladores dos países membros fizeram uma primeira proposta de harmonização dos marcos normativos existentes em cada país (Can 2002), da qual derivaram os "Principios normativos necesarios para armonizar los marcos legales y regulatórios” (Can 2002). Surpreendentemente, algumas semanas depois, os ministros de energia já tinham uma proposta de acordo e em dezembro de 2002, o Conselho Presidencial Andino ratificou o Marco General para la Interconexion Subregional de Sistemas Electricos e Intercambio Intracomunitario de Electricidad,(Decisão 536, 2002), para abrigar a execução de projetos já definidos bilateralmente por Colômbia e Equador. Contudo, o CANREL propôs suspender esse e aprovar um regime transitório capaz de abrigar as atividades existentes entre Colômbia e Equador. Esse regime transitório foi oficializado pela Decisão 720 (2009) da Comissão da Comunidade Andina e deveria durar dois anos. Esperava-se que, nesse período, fosse possível estabelecer novas bases para a revisão do Marco

15 Não estão explicitados no sítio da CAN os motivos da suspensão do acordo. 
Geral, o que não ocorreu e suscitou a necessidade de prorrogação do regime transitório (Decisão 757 2011), que deveria abrigar os projetos desenvolvidos pela Colômbia. Como discutido acima, somente em 2017 se tem um novo marco geral, voltado para regulamentar os projetos bilaterais existentes e dar base jurídica a outras iniciativas.

Por fim, há indicativos de que a CAN tenha alterado sua estratégia relativa ao setor de energia. Em 2011 criou uma estrutura institucional — SINEA — Sistema de Interconexão Energética Andino, para consolidar um corredor energético andino, ou seja, analisar a viabilidade real e as necessidades de adequação técnica para a interconexão energética de toda a sub-região andina. De conformação mais técnica, essa iniciativa recebeu recursos do Banco Interamericano de Desenvolvimento e visava subsidiar esses estudos técnicos. Contudo, a revisão do Marco Geral Decisão 536 - ainda é o objetivo a ser alcançado, sendo essa meta explicitada em todos os documentos relativos à energia no âmbito da CAN.

É interessante observar que não há, em nenhum desses acordos, a especificação do tipo de fonte de energia, se hidrelétrica, eólica, solar, dentre outras. Os negociadores provavelmente preferiram estabelecer acordos de caráter mais genérico, para abrigar as mais diversas formas de gerar energia, conforme as possibilidades dos membros dos blocos.

A partir da conformação desses Protocolos - ECOWAS e SADC - Acordo Quadro - MERCOSUL - e Marco Regulatório - CAN, pode-se avaliar os graus de regionalização/harmonização da política pública em cada um dos blocos. No caso dos dois blocos africanos, os protocolos explicitam o entendimento de que caberia aos Estados-membros viabilizar as condições para a atuação de agentes de mercado, bem como estabelecer diretrizes, através dos planos estratégicos e suas revisões, para o aumento da oferta de energia na região. Uma vez que esses Protocolos foram definidos ainda nos anos 1990, pode-se considerar que os membros entenderam que alterações não seriam necessárias, uma vez que as bases para a conformação da agenda de energia estavam suficientemente explicitadas naqueles Protocolos e os membros tinham seus interesses/preferências contemplados nesses instrumentos formais. Há, assim, uma compreensão compartilhada e estruturada ao longo de décadas de que o setor se estruturaria a partir dessas bases estabelecidas. No caso da CAN, a constituição de um Marco Geral e sua revogação logo em seguida indica que o ponto de coordenação tinha bases frágeis. Contudo, a Consolidação do Marco Regulatório em anos recentes demonstra que, depois de provavelmente anos de negociação, chegou-se ao que 
seria o formato da agenda para a região. Por fim, o MERCOSUL se limitou a um Acordo Quadro, sem regras específicas para os membros e que trata apenas de “princípios gerais” do que seria a agenda para a região.

Assim, ECOWAS e SADC apresentam um ALTO, a CAN um MÉDIO e o MERCOSUL um BAIXO nível de regionalização da agenda de energia.

\section{Estruturas Institucionais regionais e os acordos de energia elétrica}

Esta seção se propõe a identificar similaridades e diferenças nos arranjos institucionais da agenda de energia elétrica, nos quatro blocos. Com isso, busca-se evidenciar que não há um modelo a ser seguido - como o europeu, por exemplo, mas que a configuração da agenda resulta, tão somente, da coordenação dos interesses e preferencias dos países-membros, em determinada área de política, sob certas condições institucionais, o que resulta em importantes diferenças entre eles.

Os quatro blocos ratificaram, ao longo das últimas décadas, acordos relativos à energia elétrica. Esses acordos possuem diferentes características jurídicas e impactos econômicos e resultam de processos de negociação, se configurando, portanto, como o "ponto de coordenação" da agenda em cada momento histórico. Nada impede que se renegociem o formato e os termos desses acordos. Contudo, não parece ser essa a prática mais recorrente, já que somente a CAN estabeleceu nova normativa após anulação da anterior. Esse pode ser um indicativo de que: 1. o ponto de coordenação estabelecido, em alguns casos há mais de uma década, foi negociado de tal forma que não se identificou a necessidade de alteração; e/ou 2. a agenda de energia não é prioritária, não interessando aos Estados rever os termos da cooperação estabelecida.

O segundo e mais importante aspecto a ser ressaltado se refere aos termos dos acordos. Os dois blocos africanos entenderam que a cooperação entre seus membros se pautaria no sentido de regulamentar a atuação de agentes de mercado. Esse é um resultado esperado, uma vez que, sendo compostos majoritariamente por países em desenvolvimento, os investimentos necessários para viabilizar a consolidação do setor superariam a capacidade dos Estados em realizá-la. Tais Estados não dispunham das capacidades fiscais/alocativas e administrativa/de implementação requeridas para solucionar os problemas de escassez de acesso à 
energia elétrica que assolavam seus cidadãos. Ademais, fica clara a necessidade de estabelecer um marco regulatório robusto, no âmbito regional, tendo em vista o déficit de energia elétrica na região, as limitações dos Estados em resolvê-lo e o caráter estratégico deste recurso como insumo para a promoção do desenvolvimento e do bem-estar das populações desses países.

A SADC pautou suas ações na construção de estruturas institucionais, conforme estabelecido no protocolo de 1996, para conduzir a agenda. Entre os blocos analisados, a SADC é a que tem o maior número de instâncias burocráticas, a que elabora e revê planos para o setor e, mesmo contando com uma organização como a SAPP (2012), tem apresentado fracos resultados em termos de projetos “regionais" de energia elétrica. É importante observar que, na média, menos da metade da população da região da SADC tem acesso à eletricidade, sendo que na República Democrática do Congo e em Malawi, por exemplo, menos de $10 \%$ da população, ainda hoje, têm acesso à eletricidade.

Por fim, na ECOWAS, a cooperação entre os participantes visa superar as fragilidades dos países membros relativamente às capacidades de investimentos dos governos em energia diante do fato de que, na média, menos da metade da população daqueles países tem acesso à eletricidade. Os termos do Protocolo de 2003 dão as bases para a constituição desse mercado e a WAPP viabiliza essas condições, seja estruturando projetos possíveis, captando investidores e/ou gerando informações. No caso da ECOWAS, a estrutura institucional criada visa reduzir incertezas e custos de transação a que estão sujeitos os atores de mercado e regulamentar suas operações relativamente a recurso tão estratégico.

A carência de energia elétrica não constituía exatamente uma questão central para os membros dos blocos sul-americanos. Nas fronteiras entre Brasil, Argentina e Paraguai estão instaladas duas das maiores hidrelétricas do mundo - ITAIPU e Yacyretá. No caso desses dois blocos, MERCOSUL e CAN, as negociações regionais não tinham o objetivo de ampliar a oferta de energia, mas tão somente de viabilizar uma estrutura normativa capaz de abrigar eventuais atividades do setor entre seus membros. Esse é o caso das operações que já existiam entre Colômbia e Equador e entre Equador e Peru. Não se trata de criar um mercado regional de energia elétrica e, por isso, não há necessidade de estruturas institucionais que deem configuração a esse mercado. O acordo da CAN trata de regulamentar as atividades já existentes entre seus membros e para tal conta com o Comité Andino de Organismos Normativos y Reguladores de Electricidad. O MERCOSUL não estruturou instituição alguma e mantém a Reunião dos Ministros de Minas e 
Energia como fórum de discussão da agenda. Portanto, nos casos do MERCOSUL e da CAN, a questão relacionada à provisão de serviços de energia elétrica para as populações foi resolvida pelos Estados dos países que compõem os agrupamentos, de forma independente ou por meio de acordos bilaterais.

Considerando-se, então, a intercessão entre os dois eixos analíticos de regionalização e de regulação estatal, tendo em vista a empiria estudada neste artigo, a provisão de energia elétrica, pode-se localizar os pontos de coordenação dos quatro agrupamentos regionais investigados de acordo com sua distribuição na figura 2, abaixo:

Figura 2: Pontos de coordenação dos quatro agrupamentos regionais

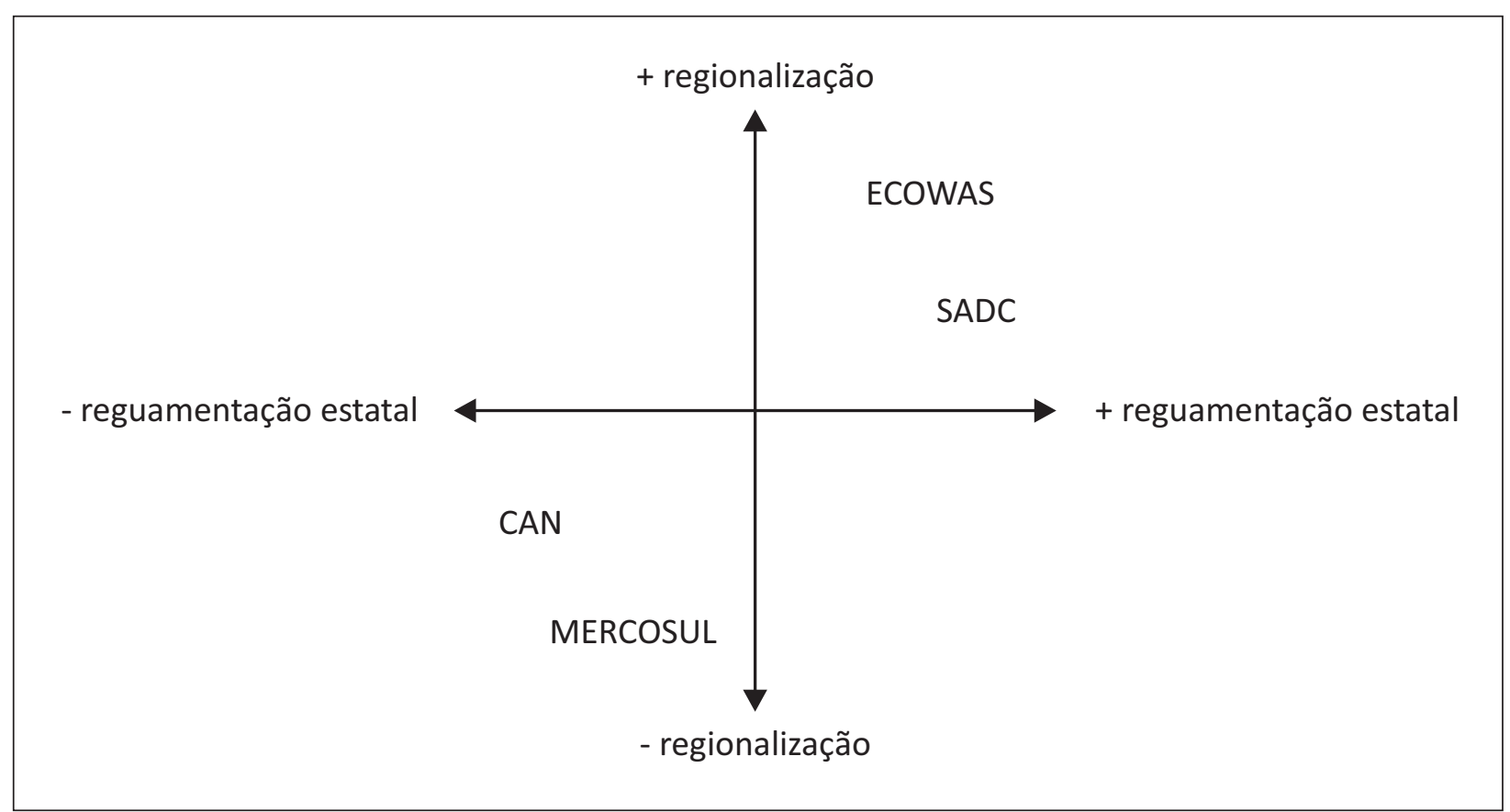

Fonte: Elaboração das autoras

\section{Considerações finais}

Este artigo apresenta um modelo analítico relativo a acordos regionais que, através da pesquisa comparada, permitisse descrever e analisar as negociações realizadas por países em desenvolvimento. O artigo se propôs a oferecer duas contribuições: a primeira e mais importante examinou como são negociadas as agendas que têm a cooperação como estratégia dominante. Há escassa literatura acerca desse dilema da ação coletiva, no qual prevalece a lógica dos jogos de coordenação. A produção tem se voltado mais para os jogos do tipo dilema do 
prisioneiro, nos quais a deserção é a estratégia dominante, na ausência de incentivos seletivos. Em contrapartida às poucas pesquisas sobre os jogos de coordenação, há diversas agendas com essa configuração negociadas diuturnamente pelos Estados, especialmente ali onde os Estados não dispõem de capacidades fiscais/ alocativas e administrativas/de implementação para garantir a provisão, de forma independente, para suas populações, de serviços essenciais, como é o caso da energia elétrica.

A segunda contribuição se refere à análise do processo de regionalização como resultado das preferências por agendas, definidas nas Cúpulas de Chefes de Estados e Governos e consolidadas em níveis burocráticos. O objetivo dos atores, neste caso, é o de converter um eixo de coordenação em um "ponto de coordenação”. Muitos analistas consideram serem essas estruturas institucionais "fracas", dada a ausência de instituições supranacionais e de mecanismos de monitoramento ou enforcement, que garantam que as políticas negociadas no âmbito regional se convertam em políticas domésticas dos Estados-membros. Buscou-se argumentar, neste artigo, que a análise da integração regional nessas agendas não precisa necessariamente se basear em critérios de "força/fraqueza" das estruturas institucionais.

Se a constituição de um acordo-quadro ou de um marco geral ou, ainda, de um protocolo for suficiente para viabilizar trocas de conhecimentos, tecnologias ou para criar bases para a consolidação de um “mercado regional” de energia, os objetivos das negociações regionais já terão sido alcançados e esse será o ponto de coordenação encontrado. $O$ artigo apresentou, ainda, um modelo para a análise de agendas temáticas específicas. Tal modelo apresenta dois eixos analíticos, o da regionalização e o da regulação estatal, como parâmetros para a análise comparativa, a partir de uma classificação composta por 3 tipos, em ambos os eixos: alta, média e baixa. Espera-se que, para além da análise da cooperação no segmento da energia elétrica, tal modelo possa contribuir para investigações de outras agendas, que foram e são negociadas por países-membros de diferentes blocos regionais. As análises relativas à convergência temporal das agendas que são negociadas no âmbito desses e de outros acordos regionais apresentam outro importante elemento para esse campo de pesquisa. As similaridades e diferenças tanto das estruturas institucionais quanto do processo de negociação das agendas que são objeto de negociação regional também podem esclarecer acerca dos objetivos e da consolidação dos acordos regionais. 


\section{Referências}

Can. 1969. Acuerdo de Cartagena. Tratados y Protocolos. Disponível em: < http://www. comunidadandina.org/Normativa.aspx?link = TP > . Acesso em: 05 jun. 2015.

Can. 2002. Marco general para la interconexion subregional de sistemas electricos e intercambio intracomunitario de electricidad. Disponível em: < http://www10.iadb. org/intal/intalcdi/PE/CM\%202013/12621.pdf > . Acesso em: 05 jun. 2015.

Cingolani, Luciana. 2013. The state of State capacity: a review of concepts, evidence and measures. Maastricht: IPD. (Working Paper Series, n. 13).

Ecowas, n.d. "Energy”. Disponível em: < https://www.ecowas.int/ecowas-sectors/energy/ > . Acesso em: 7 abr. 2020.

Ecowas. 1975. Treaty establishing the Economic Community of West African States. Lagos. Disponível em: < http://www.internationaldemocracywatch.org/attachments/351_ ecowas \%20treaty\%20of\%201975.pdf > . Acesso em: 06 jun. 2015.

Ecowas. 1993. Revised Treaty. ECOWAS COMISSION. Abuja. Disponivel em: < http://www. ecowas.int/wp-content/uploads/2015/01/Revised-treaty.pdf > . Acesso em: 06 jun. 2015

Ecowas. 1999. Forty - Fifth Session of the Council of Ministers. Lame. Disponível em: $<$ http://ecodocs.ecowas.int/?s = COUNCIL + OF + MINISTERS + 1999 > Acesso 26 set, 2016.

Ecowas. 2003. Ecowas Energy Protocol (A/P4/1/03). Disponível em: < http://www.erera. arrec.org/getattachment/3085c375-3fe8-4935-aa7d-eb6eaba923be/Documents/List/ English/ECOWAS-ENERGY-PROTOCOL.aspx > . Acesso em: 06 jun. 2015.

Ecowas. 2005. Revised Master Plan For The Generation And Transmission Of Electrical Energy, Recommendation C/REC.2/01/05. Disponível em: em < http://ugspace. ug.edu.gh/bitstream/handle/123456789/6916/2005\%2053rd\%20Session \% 20\%28Jan. pdf? sequence $=1>$. Acesso em 09 set. 2016 .

Evans, Peter. 1993. “O Estado como problema e solução.” Lua Nova Revista de Cultura e Política, n. 28-29: 107-156.

Hall, Peter A., e Taylor, Rosemary C. R. 2003. As três versões do neo-institucionalismo. Lua Nova: Revista de Cultura e Política, (58), 193-223. < https://doi.org/10.1590/S0102$64452003000100010>$.

Kurrild-Klitgaard, Peter. 1997. Rational Choice, Collective Action and the Paradox of Rebellion. Institute of Political Science, University of Copenhagen.

Locke, John. 1978. Segundo Tratado sobre o Governo. 2a ed. São Paulo: Abril Cultural. Malamud, Andres. 2011. “Conceptos, teorías y debates sobre la integración regional.” NORTEAMÉRICA. Año 6, no 2, julio-diciembre.

Martin, Lisa. 1992. “Interests, Power and Multilateralism.” International Organization, vol.46, no.4 (Autumn): 765-792. 
Martin, Lisa L e Simmons, Beth. 1998. “Theories and Empirical Studies of International Institutions.” International Organization, vol. 52, no. 4 (Autumn): 729-757.

Mello, Flavia de Campos. 1997. “Teoria dos jogos e Relações Internacionais: um balance dos debates.” Revista Brasileira de Informação Bibliográfica em Ciências Sociais, Rio de Janeiro, no. 44, $2^{\circ}$. Semestre: 105-119.

Mercosul. 1991. Tratado de Assunção. Disponível em: < http://www.tprmercosur.org/pt/ docum/Tratado_de_Assuncao_pt.pdf > . Acesso em: 10 nov. 2015.

Mercosur. 1993. Resolución 057/1993 - Directrices de Políticas Energéticas en el Mercosur. Resoluciones 1993. Disponível em: < http://gd.mercosur.int/SAM\% 5CGest Doc \% 5CPubWeb.nsf/60DF7003142CB70883257F090060A083/\$File/RES_057-1993_ ES_Direc.Pol\% C2\% A1tic.Energ.en\%20el\%20Mercosur_Acta_03_93.pdf >. Acesso em: 11 jun. 2015.

Mercosul. 2005. Acordo-quadro sobre complementação energética regional entre os Estados partes do MERCOSUL e Estados associados. Disponível em: < http://www. mercosur.int/msweb/portal \%20intermediario/Normas/acordos \%20es/44_05_ ACUERDO \% 20MARCO \% 20COMPLEMT \% 20ENERGETICA \% 20MCS-EA_ES.pdf > . Acesso em: 11 jun. 2015.

Mercosur.1996. "Resolución 150/1996 - Pautas Negociadoras del Subgrupo de Trabajo No 9” - Energía. Resoluciones 1996. Disponível em: < http://gd.mercosur.int/SAM \% 5CGestDoc \% 5CPubWeb.nsf/56BAC175E63641F083257F090060E470/\$File/ RES_151-1996_PT_SGT11.pdf >. Acesso em: 11 jun. 2015.

Mercosur.1998. "Memorandum de Entendimiento Relativo a los Intercambios Eléctricos e Integración Eléctrica en el Mercosur.” Decisiones 1998. Disponível em: < http:// www.mercosur.int/innovaportal/v/2930/2/innova.front/decisiones-1998 > . Acesso em: 11 jun. 2015.

Mercosur. 2000. “Reunión de Ministros de Minas y Energía del Mercosur” Decisiones 2000. Disponível em: < http://www.mercosur.int/innovaportal/v/3189/2/innova. front/decisiones-2000 > . Acesso em: 11 jun. 2015.

Olson, Mancur. 1999. A Lógica da Ação Coletiva. São Paulo: EDUSP.

Sadc, n.d. "Directorates \& Units”. Disponível em: < https://www.sadc.int/sadc-secretariat/ directorates/ > . Acesso em: 7 abr. 2020.

Sadc. 1996. Consolidated text of Treaty of the Southern African Development Community. Disponível em: < http://www.sadc.int/files/5314/4559/5701/Consolidated_Text_of_ the_SADC_Treaty_-_scanned_21_October_2015.pdf. Acesso em: 05 out. 2015.

Sadc. 1996. Protocol on Energy - 1996. Disponível em: < http://www.sadc.int/files/3913 /5292/8363/Protocol_on_Energy1996.pdf > . Acesso em: 05 out. 2015.

Sadc. 2001. Extraordinary Summit Communiqué Namibia - Windhoek: disponívem em < http://www.sadc.int/files/1613/7243/4333/SADC_ES_Report_2011-2012_web. pdf $>$. Acesso em 09.set. 2016. 
Sadc. 2012. The SADC regional infrastructure Master Plan. Disponível em: < http://www. sadc.int/files/5413/5293/3528/Regional_Infrastructure_Development_Master_Plan_ Energy_Sector_Plan.pdf > . Acesso em: 05 out. 2015 .

Sapp.2012. "Energising the region for economic development". Monthly Report. November 2012 Issue No. R11-12. Disponível em:<http://www.sapp.co.zw/docs/R11-\%20 November \% 202012\%20SAPP\% 20Monthly \% 20Report \% 20(2).pdf > . Acesso em: 05 out. 2015.

Schmitter, Phillipe.2010. “A Experiência da Integração europeia e seu potencial para a integração regional.” Lua Nova, N. 80: 9-44.

Snidal, Duncan. 1985. “Coordination versus Prisoners’ Dilemma: Implications for International Cooperation and Regimes." American Political Science Review, 79(4), 923-942. doi:10.2307/1956241.

Stein, Arthur. 1982. "Coordination and Collaboration: Regimes in an Anarchic World.” International Organization, 36(2), 299-324. Retrieved April 7, 2020, from $<$ www.jstor.org/stable/2706524 > .

Wapp. 1999. "Annual Activity Report Of The Wapp Secretary General.” ECOWAPP. Disponível em: < http://www.ecowapp.org/?page_id = 372. Acesso em: 06 out. 2015

World Bank. n.d. “World Development Indicators. DataBank.” Acesso em: 7 abr. 2020. Disponível em: < https://databank. worldbank.org/reports.aspx? source $=2 \&$ series $=$ EG. ELC.ACCS.ZS\&country $=>$. 